\title{
Valoración pragmática del lenguaje en niños con cromosomopatias de baja prevalencia
}

\author{
MAITE FERnÁNDEZ-URQUIZA \\ Universidad de Oviedo \\ Facultad de Filosofia y Letras \\ C/ Amparo Pedregal s/n \\ 33011 Oviedo (España) \\ E-mail: fernandez- \\ maite@uniovi.es
}

\section{VALORACIÓN PRAGMÁTICA DEL LENGUAJE EN NIÑOS CON CRO- MOSOMOPATÍAS DE BAJA PRE- VALENCIA}

RESUMEN: Las cromosomopatías consisten en duplicaciones o deleciones de fragmentos cromosómicos que suelen conllevar alteraciones del lenguaje. Debido a su baja prevalencia, no se dispone aún de descripciones precisas del déficit lingüístico que llevan asociado ni de caracterizaciones funcionales del lenguaje de los afectados. En este trabajo proponemos una metodología pragmático-funciona para la valoración del déficit lingüís tico en niños con cromosomopatías de baja prevalencia, que vincula las alteraciones detectadas en los componentes estructurales del lenguaje con las disfunciones comunicativas que generan. Discutimos la problemática que plantea la transcripción y codificación de la muestra con las herramientas del proyecto CHILDES y su etiquetado con PREP-CORP, ejemplificando nuestras reflexiones con datos del corpus CHROMOLANG. Concluimos que el análisis pragmático de muestras de lenguaje oral no elicitado complementa la evaluación mediante pruebas estandarizadas, contribuye al diagnóstico y permite orientar la intervención logopédica hacia el éxito comunicativo.

PALABRAS CLAVES: cromosomopatías; pragmática; déficit lingüístico; CHILDES; PREP-CORP.

SUMARIO: 1. Introducción. 2. Metodologia: Análisis pragmático de lenguaje oral conversacional. 2.1. Recogida de la muestra. 2.2. Transcripción y codificación de la muestra. 2.3. Etiquetado pragmático. 3. El déficit pragmático en niños con cromosomopatías de baja prevalencia. 3.1. Déficit pragmático específico. 3.2. Déficit pragmático de base gramatical. 3.3 . Conductas compensatorias. 4. Conclusiones.

\author{
Ma SALUd Jiménez-ROMERO \\ Universidad de Córdoba \\ Facultad de Ciencias de la \\ Educación \\ C/ San Alberto Magno s/n \\ 14004 Córdoba (España) \\ E-mail: m12jirom@uco.es
}

PRAGMATIC ASSESSMENT OF LANGUAGE IN CHILDREN WITH LOW PREVALENCE CHROMOSOMOPATHIES

ABSTRACT: Chromosomopathies consist of duplications or deletions of chromosomal fragments that usually produce language impairment. However accurate descriptions of structural and functional linguistic deficits caused by chromosomopaties are not yet available due to the low prevalence of these alterations. Our aim in this paper is to put forward a pragmatic methodology in order to assess linguistic impairment in children with low prevalence chromosomopathies. This method links structura linguistic deficits together with the prag matic dysfunctions they cause in communication. We discuss the transcrip tion and coding of the data with the CHILDES tools and its labeling with PREP-CORP, and we illustrate our reflections with examples from the CHRO MOLANG corpus. We conclude that the pragmatic analysis of natural oral language data complements the traditiona assessment through standardized tests, contributes to diagnosis, and allows speech-language clinicians to focus on strategies to achieve communication success during the intervention.

KEY WORDS: chromosomopathies; pragmatics; linguistic impairment; CHILDES; PREP-CORP.

SUMMARY: 1. Introduction. 2 Method: Pragmatic analysis of conversational oral language. 2.1. Data collection. 2.2. Data transcription and coding. 2.3. Pragmatic labeling. 3. Pragmatic impairment in children with low prevalence chromosomopathies. 3.1. Specific pragmatic deficit 3.2. Grammar-based pragmatic deficit. 3.3. Compensatory behaviours. 4 Conclusions.

\author{
ANTONIO BENÍtez-BURRACo \\ Universidad de Sevilla \\ Facultad de Filología \\ C/ Palos de la Frontera s/n \\ 41004 Sevilla (España) \\ E-mail: abenitez8@us.es
}

\section{ÉVALUATION PRAGMATIQUE DU LANGAGE CHEZ LES ENFANTS PRESENTANT DES ANOMALIES CHROMOSOMIQUES DE FAIBLE PREVALENCE}

RÉSUMÉ: Les anomalies chromosomiques consistent en des duplications ou des délétions de fragments chromosomiques impliquant généralement des altérations du langage. En raison de sa faible prévalence, il n'existe toujours pas une description précise du déficit linguistique des individus affectés ni de caractérisation fonctionnelle de leur langage. Dans cet article, nous proposons une méthodologie pragmatique et fonctionnelle pour l'évaluation du déficit linguistique chez les enfants atteints d'anomalies chromosomiques à faible prévalence, qui relie les altérations détectées dans les composants structurels du langage avec les dysfonctionnements de la communication. Nous discutons les problèmes posés par la transcription et le codage de l'échantillon avec les outils du projet CHILDES et son étiquetage avec PREP-CORP, illustrant nos réflexions avec les données du corpus CHROMOLANG. Nous concluons que l'analyse pragmatique d'échantillons de langage oral naturel complète l'evaluation au moyen de tests standardisés, contribue au diagnostic et permet d'orienter l'intervention en orthophonie vers le succès de la communication.

MOTS CLÉS: anomalies chromosomiques; pragmatique; déficit linguistique; CHILDES; PREP-CORP.

SOMMAIRE: $1 . \quad$ Introduction. 2 Méthodologie: Analyse pragmatique du langage oral conversationnel. 2.1 Collection de l'échantillon. 2.2. Transcription et codage de l'échantillon. 2.3. Étiquetage pragmatique 3. Le déficit pragmatique chez les enfants présentant des anomalies chromosomiques de faible prévalence. 3.1. Déficit pragmatique spécifique. 3.2. Déficit pragmatique de base grammaticale. 3.3. Comportement compensatoire. 4. Conclusions. $\begin{array}{ll}\text { Fecha de Recepción } & 05 / 02 / 2019 \\ \text { Fecha de Revisión } & 07 / 03 / 2019 \\ \text { Fecha de Aceptación } & 15 / 05 / 2019 \\ \text { Fecha de Publicación } & 01 / 12 / 2020\end{array}$
DOI: http://doi.org/ 10.25267/Pragmalinguistica.2020.iextra2.09 


\title{
Valoración pragmática del lenguaje en niños con cromosomo- patias de baja prevalencia
}

\author{
Maite FernándeZ-Urquiza, Ma SAlud JiméneZ-Romero \& ANTonio Benítez-BurRaco
}

\section{INTRODUCCIÓN}

Las cromosomopatías consisten en duplicaciones o deleciones de fragmentos cromosómicos de tamaño variable que suelen conllevar alteraciones del lenguaje. Debido a su baja prevalencia y a la heterogeneidad de las disfunciones lingüísticas que pueden ocasionar las deleciones y duplicaciones de distintos fragmentos cromosómicos, no existen todavia descripciones precisas del déficit lingüístico que llevan asociado. Su evaluación se realiza normalmente mediante pruebas estandarizadas que, necesariamente, proporcionan una información poco detallada sobre el perfil lingüístico de esta población clínica. A lo anterior se suma la ausencia total de caracterizaciones funcionales del lenguaje de estos niños, lo que dificulta tanto el diagnóstico como el diseño de programas de intervención logopédica específicos. En efecto, es habitual que, para cada caso, se diseñe una intervención basada en estrategias que han resultado de utilidad en casos anteriores con un perfil semejante en los test genéricos, a pesar de no ser similares desde el punto de vista genético, olvidando además que puntuaciones similares en estas pruebas pueden esconder diferencias cognitivas importantes: "sometimes equivalent behavioral scores camouflage very different cognitive processes" (Donnai y Karmiloff-Smith, 2000: 167).

En este trabajo proponemos una metodologia pragmático-funcional para la descripción sistemática y exhaustiva del déficit lingüistico en niños con cromosomopatías de baja prevalencia, que vincula las alteraciones detectadas en los componentes estructurales del lenguaje con las disfunciones comunicativas que generan, con el objetivo de contribuir al diseño de intervenciones tempranas específicas para los niños afectados por cromosomopatias diversas, que previsiblemente presentarán perfiles lingüísticos y cognitivos divergentes.

\section{METOdOLOGÍA: ANÁlisis PRAGMÁtico DE LENGUAJE ORAL CONVERSACIONAL}

Como acabamos de señalar, las baterías de test estandarizadas son un instrumento de uso generalizado para la evaluación del déficit lingüístico en niños con cromosomopatías de baja prevalencia, tanto en el ámbito logopédico como neuropsicológico. Estos test proponen pruebas descontextualizadas que resultan útiles para detectar alteraciones concretas en los componentes estructurales clásicos del lenguaje (fonología, morfología, sintaxis, léxico).

Ahora bien, en palabras de Muñoz-Céspedes y Melle (2004: 854): "El empleo de las baterias tradicionales encubre las dificultades (...) para utilizar 
el lenguaje en contextos interactivos y comunicativos, ya que estas pruebas miden habilidades lingüisticas de forma aislada, que raramente aparecen como tal en el contexto de la vida diaria". En efecto, para llegar a conocer el estado real de lenguaje de estos niños es necesario realizar también una valoración funcional, es decir, del uso que son capaces de hacer de sus recursos verbales preservados. Solo de este modo podremos valorar su competencia comunicativa o, en otras palabras, si son capaces de utilizar las habilidades verbales y no verbales de que disponen para interactuar adecuadamente en contextos naturales. Como es obvio, una evaluación de este tipo solo puede hacerse por medio del análisis pragmático de datos con validez ecológica, es decir, de muestras de lenguaje oral no elicitado, sino producido de manera natural por el niño en situaciones cotidianas con interlocutores familiares (Diaz, 1995; Fernández-Urquiza et al., 2015a; GallardoPaúls, 2007, 2009). Se trata de una metodología que bebe de la aproximación cualitativa al análisis lingüístico que se encuentra ya en los estudios pioneros de Jakobson (1935, 1941, 1956), Lesser (1978) y Crystal (1981, 1982) en el ámbito de la Lingüística Clínica. En esta aproximación cualitativa y funcional al análisis del déficit, las manifestaciones lingüísticas se conciben como fenómenos inextricablemente ligados a sus usos comunicativos, es decir, como conductas para cuya valoración es necesario tener en cuenta el contexto en que se producen, así como las intenciones comunicativas del hablante y las funciones sociales que pretende satisfacer.

En otras palabras, al evaluar el déficit comunicativo estamos evaluando una dimensión ligada a otras que trascienden lo puramente verbal, y de ahi el énfasis en la recogida de datos naturales registrados en vídeo, que permitan observar paralelamente las conductas comunicativas paraverbales y no verbales de los informantes. Tomar en cuenta esta estructura triple básica de la comunicación (Poyatos, 2003) resulta imprescindible de cara a diseñar estrategias de intervención optimizadas para una población clínica que manifiesta una clara voluntad interaccional, y que comunica mucho más de forma no verbal de lo que consigue expresar verbalmente. En este sentido, conocer las habilidades comunicativas paraverbales y no verbales que los niños con cromosomopatias de baja prevalencia utilizan de manera espontánea resulta fundamental.

\subsection{RECOGIDA DE LA MUESTRA}

La combinación de evaluaciones de tipo formal y funcional requiere de la colaboración de profesionales de los ámbitos de la Lingüística y la Logopedia. Así, será el logopeda de los niños quien solicite a sus tutores la grabación en vídeo de fragmentos de conversación cotidiana en su propio domicilio o en entornos naturales para los informantes, que nos permitan observar cómo se desenvuelven espontáneamente con sus familiares cercanos, como sus padres, hermanos y abuelos. Si el logopeda se encuentra lo suficientemente familiarizado con los niños y sus familiares, hasta el punto de que su 
presencia no inhibe ya la interacción natural que se produce entre ellos, es posible utilizar también para el análisis conversaciones en las que el logopeda tenga una observación participante.

Para la realización de esta investigación hemos reunido una media de 15 minutos de muestra de cada uno de los casos que hemos estudiado, y asi estamos construyendo un corpus que hemos denominado CHROMOLANG (Chromosomopathies and Language) que, cuando esté terminado, contará con aproximadamente 300 minutos de muestra correspondientes a 20 sujetos.

\subsection{TRANSCRIPCIÓN Y CODIFICACIÓN DE LA MUESTRA}

La transcripción y la codificación de la muestra se han realizado con las herramientas del proyecto CHILDES (CHId Language Data Exchange System), coordinado en sus inicios por Brian MacWhinney y Catherine Snow (MacWhinney y Snow, 1985, 1990). Como señalan Diez-Itza, Snow y MacWhinney, 1999: 517), CHILDES es un sistema que "proporciona potentes herramientas para investigar en el marco de la metodología de Registro, Transcripción y Análisis de Muestras de Habla Espontánea (RETAMHE)".

CHILDES se encuentra actualmente integrado en el proyecto Talkbank (www.talkbank.org), en el que se incluyen bases de datos no solo de lenguaje infantil típico en diversas lenguas, sino también bases de datos clínicos, que recogen muestras de lenguaje oral de adultos con demencia, lesionados de hemisferio derecho, traumatismo craneoencefálico, afasia, así como de niños y adultos con trastornos de espectro autista y disfemia. En cualquier caso, el objetivo inicial del proyecto CHILDES, que se mantiene en la actualidad en las distintas bases de datos de Talkbank, es compartir muestras de lenguaje oral en un formato de archivo unificado (.cha) que utiliza, además, un formato de transcripción y codificación estandarizado que se llama CHAT (Codes for the Human Analysis of Transcripts).

La elección de este formato para CHROMOLANG se debe, por una parte, a la exhaustividad y versatilidad de la codificación y del etiquetado que posibilita CHAT, así como a la variedad de análisis automatizados que permite implementar CLAN (Computerized Language ANalysis), el paquete de software que Talkbank pone gratuitamente a disposición de la comunidad internacional de investigadores. Por otra parte, nuestro objetivo es que CHROMOLANG forme parte de Talkbank a medio plazo, contribuyendo así a crear una base de datos de lenguaje oral de niños con cromosomopatías de baja prevalencia, sobre la que puedan hacerse análisis ulteriores del lenguaje en este tipo de trastornos.

CHAT permite realizar una codificación paralela a la propia transcripción de los datos, de manera que las líneas principales no son un simple volcado, sino que en ellas se introduce ya información que orientará el etiquetado posterior (Fernández-Urquiza y Gallardo-Paúls, 2015b). Así, por ejemplo, en 
las lineas principales pueden codificarse fenómenos relativos a la articulación (por ejemplo, si es silabeada o esforzada), a la fluidez (si hay disfluencias, repeticiones o reformulaciones), a la duración de las pausas, además de incluir otras informaciones de tipo no verbal como, por ejemplo, el uso comunicativo de la mirada, o el uso de gestualidad emblemática, ilustradora o reguladora (Ekman y Friesen, 1981), como se observa en el ejemplo siguiente:

${ }^{*} \mathrm{CHI}$ :

mi didicha [*] [=! syllabified] y cuando \&do la ido $\uparrow[*]($.$) señala <$ al de la $>$ [/] $<$ al de la $\left.>{ }^{*}\right]$ izquierda ", no [=! looks MOT in the eyes]?

\%err: didicha=derecha \$PHO; ido=giro \$PHO; al de la=a la \$SYN \$MOR

Simultáneamente, los errores que produce el niño se señalan con un asterisco en la línea principal. A continuación, en la línea dependiente de error se procede a desambiguar el target (es decir, se identifica lo que el informante quiso decir, cuando es posible) y se clasifica el error como de tipo fonológico, morfológico, sintáctico o léxico. Esta clasificación constituye la base de etiquetados posteriores más pormenorizados que pueden realizarse para cada uno de esos niveles, pero cuyo alcance excede los límites y el propósito de este trabajo.

Es importante señalar la trascendencia que tiene esta primera codificación con CHAT, y reflexionar sobre las dificultades que pueden presentarse durante el proceso. Así, por ejemplo, los informantes del corpus CHROMOLANG son niños procedentes de distintas regiones de Andalucía, por lo que resulta fundamental atender a sus particularidades dialectales. Para ello, es imprescindible prestar atención al modo en que sus progenitores y familiares cercanos realizan ciertos fonemas (fundamentalmente /s/, /1/, / / , / r /) que suelen desaparecer, aspirarse o asimilarse en margen postnuclear silábico, dependiendo del contexto fónico, y cuya omisión puede dar lugar o no a un aumento de la apertura de la vocal precedente. En consecuencia, durante la transcripción de la muestra hemos procurado no codificar como erróneas ciertas realizaciones del informante que se desvian del estándar y que sus progenitores o familiares cercanos producen de manera sistemática, como se observa en el siguiente fragmento, en el que tanto la informante como su madre y su hermano omiten los sonidos fricativos alveolares y vibrantes en final de palabra:

*BOY: $\quad$ Ana $\ddagger$ qué quieres $[=!$ deleted $/ \mathrm{s} /$, dialectal] que te regale ?

${ }^{*} \mathrm{CHI}$ :

1 (o) que tú quieras [=! deleted /s/, dialectal] [=! raises her arm toward BOY]

*BOY: lo que yo quiera no: lo que $\mathrm{xxx} \mathrm{xxx}$.

${ }^{*} \mathrm{CHI}: \quad 1(\mathrm{o})$ que tú quieras $[=!$ deleted $/ \mathrm{s} /$, dialectal] [=! claps once, showing EBM toward BOY]!

*MOT: $\quad$ y dónde vas $[=!$ deleted $/ \mathrm{s} /$, dialectal $]$ a dormir $[=!$ deleted $/ \mathrm{r} /$, dialectal] ?

Otro problema con el que podemos encontrarnos a la hora de transcribir y codificar la muestra es el de la falta de inteligibilidad. El ejemplo que aca- 
bamos de proporcionar no es en absoluto representativo del grado de inteligibilidad que se observa normalmente en las producciones verbales de los informantes de CHROMOLANG que, por el contrario, suele ser muy bajo. Esta falta de inteligibilidad puede venir ocasionada por dislalia, disglosia, apraxia del habla, conciencia fonológica poco desarrollada, alteraciones de la linea melódica causadas por falta de control del volumen de la voz, disfemia, etc. En todos estos casos, cuando el habla, además de estar articulatoriamente deformada, se produce de manera disfluente y fragmentada, puede resultar muy dificil diferenciar lo que pretende ser una palabra de lo que simplemente es una disfluencia, como ocurre en el ejemplo siguiente, donde las disfluencias se señalan mediante el uso del ampersand (\&):

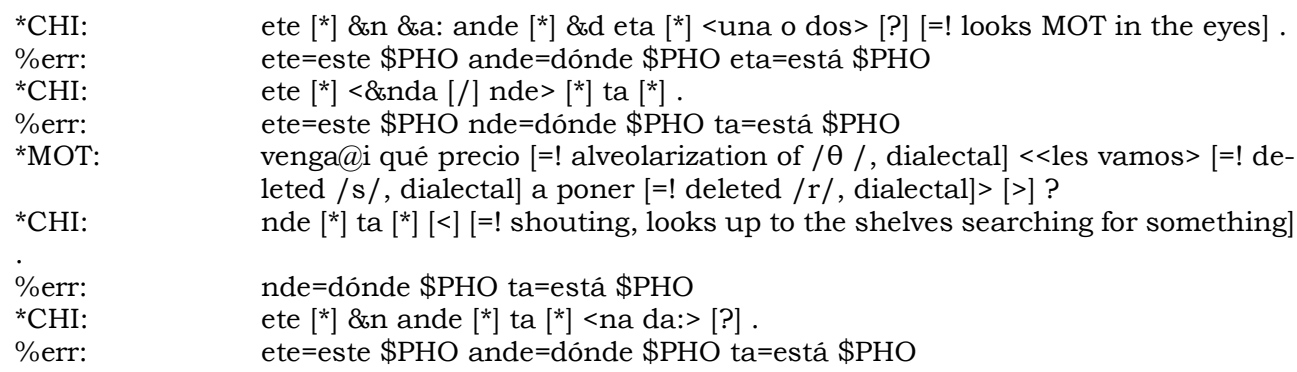

Una codificación adecuada de estos fenómenos resulta de vital importancia, ya que de ello dependerá el cálculo de la longitud media de enunciado en palabras (MLUw), lo que a su vez nos permitirá hacernos una idea del grado de desarrollo morfosintáctico que el informante exhibe en contextos naturales.

\subsection{ETIQUETADO PRAGMÁtICO}

A las lineas principales de la transcripción, que recogen y codifican los enunciados de los informantes, y a las lineas de error, que desambiguan esos errores y los categorizan, hay que añadir aún una tercera línea en la que se recoge el etiquetado pragmático de los enunciados.

Como señala Verschueren (1999), cuando se evalúa el lenguaje en uso, todo fenómeno lingüístico ha de ser considerado desde una perspectiva pragmática. En el ámbito clínico, Fernández Pérez (2000: 114) hace notar que

la naturaleza de los hechos de lengua no se explica sin contemplar su ontología pragmática, o lo que es lo mismo, su carácter interpersonal e interactivo. Así que, en términos metodológicos, si queremos abordar los fenómenos lingüísticos en su materialidad comunicativa, la perspectiva ha de ser -inexcusablemente- pragmática. De modo más específico (...), son las situaciones lingüísticas problemáticas y deficitarias las que en mayor medida exigen la adopción del prisma pragmático [...]. 
Por otra parte, y puesto que el uso natural del lenguaje demanda el concurso de funciones mentales superiores de carácter no específicamente lingüístico (a saber, capacidad metarrepresentacional, habilidades inferenciales, inhibición, atención, etc.), un estudio de orientación pragmática puede contribuir a detectar, confirmar, o descartar otros problemas cognitivos en niños con cromosomopatias de baja prevalencia, al igual que lo hace en adultos con daño cerebral adquirido (Davis, 2007).

Así pues, una vez clasificados los errores estructurales en el lenguaje oral del informante, valoramos sus efectos comunicativos y los etiquetamos mediante PREP-CORP (Fernández-Urquiza et al., 2017) un sistema de etiquetado pragmático de corpus clínicos orales desarrollado en el grupo LOGIN de la Universidad de Oviedo en el marco del Proyecto SYNDROLING (DiezItza et al., 2014) a partir del Protocolo Rápido de Evaluación Pragmática-Revisado (PREP-R, Fernández-Urquiza et al., 2015a). PREP-CORP está disenado para trabajar con el paquete de programas CLAN, que permite hacer recuentos automáticos de frecuencias, calcular la longitud media de enunciado (MLU), la type-token ratio (TTR), así como distintas búsquedas de palabras aisladas o en contexto, adaptadas siempre a los fines de la investigación (MacWhinney, 2000). Como ya hemos comentado, CLAN trabaja con archivos en formato CHAT (.cha). Una caracteristica muy interesante de estos archivos es que se pueden añadir lineas dependientes a los enunciados del informante. Así, por ejemplo, la línea \%sit introduce información que describe la situación comunicativa, y se usa cuando el transcriptor considera que eso facilita la comprensión del enunciado (MacWhinney, 2000: 83):

\footnotetext{
"*EVE: $\quad$ what that?

*EVE: $\quad$ woof@o woof@o.

\% sit: dog is barking"
}

Además, estas lineas dependientes pueden adaptarse a los propósitos de la investigación, es decir, podemos crear nuevas líneas añadiendo el símbolo $\% x$ antes de las tres letras que dan nombre a la linea. Para el etiquetado pragmático con PREP-CORP hemos creado tres lineas dependientes que se corresponden con los tres niveles de análisis pragmático que propone el PREP-R.

\begin{tabular}{|l|l|l|}
\hline Niveles pragmáticos & Enunciativo & \%xepr (Enunciative PRagmatics) \\
\cline { 2 - 3 } & Textual & \%xtpr (Textual PRagmatics) \\
\cline { 2 - 3 } & Interactivo & \%xipr (Interactive PRagmatics) \\
\hline
\end{tabular}

Cuadro 1: Niveles de análisis pragmático en PREP-R y PREP-CORP

El etiquetado que hemos realizado en el corpus CHROMOLANG es el del nivel pragmático enunciativo (\%xepr) que se estructura a su vez en torno a tres subniveles de análisis (actos de habla, tareas de edición, e inferencias). El simbolo $\$$ seguido de las letras identificadoras de subnivel precede a un número que indica la correspondencia con uno de los ítems del PREP-R: 


\begin{tabular}{|c|c|c|}
\hline \multirow{2}{*}{$\begin{array}{c}\text { Pragmática Enunciativa } \\
\text { \%xepr }\end{array}$} & Actos de Habla & \$sa2 (Speech Acts) \\
\cline { 2 - 3 } & Tareas de Edición & \$et3, \$et4 (Editing Tasks) \\
\cline { 2 - 3 } & Inferencias & \$i5, \$i6 (Inferences) \\
\hline
\end{tabular}

Cuadro 2: Subniveles de la Pragmática Enunciativa y sus etiquetas en PREP-CORP

Estos tres subniveles nos permiten valorar: 1) si el informante es capaz de comprender actos de habla indirectos; 2) si demuestra conciencia metapragmática y/o utiliza estrategias compensatorias de sus dificultades expresivas, y 3) si es capaz de realizar contribuciones conversacionales acordes a las máximas del Principio de Cooperación de Grice (1975).

Puesto que los tres subniveles contienen en su conjunto un total de 68 etiquetas (10 etiquetas principales, 47 secundarias, y 11 opcionales) que además pueden combinarse de maneras diversas entre sí, abarcando un número realmente muy amplio de fenómenos pragmáticos, utilizaremos los datos del corpus CHROMOLANG para ilustrar los fenómenos pragmáticos más representativos en niños con cromosomopatias de baja prevalencia. Al hacerlo, focalizaremos nuestra atención no solo en los déficits pragmáticos que presentan estos niños, sino también en su voluntad de contribuir al intercambio a pesar de sus escasos recursos expresivos.

\section{El DÉFICIT PRAGMÁTICO EN NIÑOS CON CROMOSOMOPATÍAS DE BAJA PREVA- LENCIA}

\subsection{DÉFICIT PRAGMÁTICO ESPECÍFICO}

Las transgresiones de las máximas de calidad (MQL $\left.{ }^{1}\right)$ y relación (MRL) (Grice, 1975) son posiblemente una de las formas más llamativas en que se manifiesta el déficit pragmático. En el corpus CHROMOLANG encontramos numerosos enunciados en que los informantes transgreden estas máximas, aunque por razones diversas.

En algunos casos, las transgresiones se producen cuando el niño tiene problemas para comprender una tarea o alguna explicación que le proporciona su madre. Esto es lo que ocurre en el caso de CHROMOLANG_1, un niño de 5;8 años en el momento de la grabación, que presenta una microduplicación en el segmento 1q21.1 (Benitez-Burraco et al., 2018). En el siguiente fragmento de conversación ${ }^{2}$, la madre propone al niño clasificar los coches por modelos en lugar de hacerlo por colores, como habian estado haciendo hasta ese momento:

*MOT: míralo (.) mira el modelo [/] el modelo (.) no el color (.) sino el modelo .

*CHI: a ver@i [=! looks attentively to the car and searchs for a similar one on the table].

*MOT: yo creo que \&e este <se le pa> [/-] se le puede parecer mucho „no ?

\footnotetext{
${ }^{1}$ Las letras mayúsculas se corresponden con las etiquetas del PREP-CORP para cada uno de los fenómenos mencionados.

2 En todos los ejemplos hemos eliminado las codificaciones relativas a las particularidades dialectales de pronunciación para facilitar la legibilidad.
} 
${ }^{*}$ CHI: no [=! scratchs his head, then shakes head no].

\%xepr: \$i5:MQL:ASW:ICOM

*MOT: sí: ponlo al la(d)o@d (.) mira@i .

${ }^{*} \mathrm{CHI}: \quad<$ en \&e \&e \&e> $[>]$.

*MOT: ponlo al la(d)o@d [<] (.) pon el azul al la(d)o@i.

${ }^{*} \mathrm{CHI}: \quad 0$ [=! CHI puts the blue car close to the one MOT is pointing to]. [+ trn]

*MOT: se parecen?

${ }^{*} \mathrm{CHI}: \quad$ no [=! shakes head no].

\%xepr: \$i5:MQL:ASW:ICOM

La nueva tarea parece ser demasiado compleja para el niño, de ahí que hayamos señalado en el etiquetado que la transgresión de calidad se produce por incomprensión (ICOM). El niño comete también transgresiones de relación en forma de cambios bruscos de tema. En el siguiente ejemplo, nuestro informante pregunta a su madre por el significado de una señal de tráfico de juguete, pero pronto pierde interés (posiblemente porque no comprende las explicaciones que ella le proporciona) y decide reconducir la conversación hacia sus intereses:

*MOT: vaya@i esa es dirección única (.) un único sentido.

${ }^{*} \mathrm{CHI}$ : mi didicha [*] [=! syllabified] y cuando \&do la ido $\uparrow\left[{ }^{*}\right]($.) señala $<$ al de la $>$ [/] <al de la $>$ $\left[{ }^{*}\right]$ izquierda "no [=! looks MOT in the eyes]?

\%err: didicha=derecha \$PHO; ido=giro \$PHO; al de la=a la \$SYN \$MOR

*MOT: y cuando la giras pallá@d ?

${ }^{*} \mathrm{CHI}$ e:s [=! points to the toy looking MOT in the eyes] (.) nanasión [*] [=! effortful articulation, syllabified, regulates speech rhythm with movements of his head] prohibida .

\%err: $\quad$ nanasión=dirección \$PHO

\%xepr: \$i5:MQL:ASW:ICOM

*MOT: no: cuando giras pallá@d es un sentido único (.) una dirección única (.) y pallá@d otra dirección única (.) una xxx xxx si le das la vuelta (.) señala pa@d la derecha: xxx [>].

${ }^{*} \mathrm{CHI}: \quad$ pre:pa(r)dos $\uparrow($.$) li:sto:s \uparrow$ (.) ya: [=! syllabified, looking at MOT]!

\%xepr: \$i5:MRL:RPT:NRL

*MOT: y quién va a hacer la carrera ?

En efecto, muchos de los enunciados de CHROMOLANG_1 evidencian problemas de comprensión, lo que se manifiesta en una frecuencia de transgresiones de las máximas de relación y de calidad mucho mayor que la que se observa en niños con desarrollo típico (en adelante, DT). Así, por ejemplo, un niño con DT de 5 años de edad comete una media de 10,4 transgresiones de relación por cada mil tokens de muestra (Fernández-Urquiza et al., 2015c), mientras que CHROMOLANG_1 comete 13 en una muestra de tan solo 327 tokens (es decir, unas 40 transgresiones por cada mil tokens). En el caso de las transgresiones de calidad, la media para los niños con DT es de 11,2 por cada mil tokens, mientras que nuestro informante comete 9 en una muestra de 327 tokens (27,5 por mil tokens).

Las transgresiones de calidad pueden deberse también a conductas impulsivas o inmediatas (IMM), es decir, a la falta de inhibición del informante, así como a razonamientos aberrantes que evidencian que el niño se encuentra cognitivamente desnivelado con respecto a sus interlocutores, es decir, que no comparte el mundo posible de referencia (WOR). En cualquier caso, 
la combinación del análisis pragmático del lenguaje oral espontáneo del informante con los resultados de test normalizados para la evaluación de sus capacidades cognitivas y ejecutivas resulta fundamental para poder interpretar adecuadamente sus conductas y completar su diagnóstico.

Este es el caso de CHROMOLANG_2, un niño de 11;6 años en el momento de la grabación, con una microdeleción en 8 p23.1 y una microduplicación en 9 p24.3. El siguiente ejemplo se ha extraído de una conversación en la que el niño juega con su madre a las adivinanzas. Cada uno escoge una tarjeta con un dibujo que ha de mostrar al otro, pero que él mismo no puede ver. A partir de ese momento, tienen que hacerse preguntas para averiguar qué es lo que hay dibujado en su tarjeta. La madre del niño ha conseguido averiguar que en su tarjeta hay una fruta, pero no sabe cuál. La fruta en cuestión es la piña. A continuación, se muestran las respuestas que el niño proporciona a las preguntas de su madre:

*MOT: e:h es de color amarillo ?

${ }^{*} \mathrm{CHI}$ : no [=! shakes head no].

\%xepr: \$i5:MQL:ASW:IMM

(...)

${ }^{*}$ MOT: y tiene pepitas ?

${ }^{*} \mathrm{CHI}: \quad$ zí $[*]$.

\%err: $\quad z i=$ sí \$PHO

\%xepr: \$i5:MQL:ASW:WOR

En el primer caso, etiquetamos la respuesta como inmediata o impulsiva (IMM), es decir, una respuesta que el niño proporciona sin reflexionar, porque la rectifica un par de turnos después. Sin embargo, la segunda respuesta es claramente un WOR, es decir, no se corresponde con el estado de cosas del mundo real.

El tipo de preguntas que hace el niño cuando llega su turno en el juego también pone de manifiesto tanto su falta de inhibición como el carácter anómalo de sus razonamientos:

*CHI: e:s (.) [/] es u:n animal ?

*MOT: sí [=! nods].

*CHI: o:h@i [=! smiles] e:s [/-] (.) hu:m@i tie:ne: [/] tiene cola?

*MOT: sí [=! nods] .

${ }^{*} \mathrm{CHI}$ : un león [=! looks MOT in the eyes smiling].

\%xepr: \$i5:MQL:QST:WOR:IMM

Como se puede observar,CHROMOLANG_2 aventura una hipótesis sobre el dibujo de su tarjeta cuando dispone tan solo de dos semas muy inespecíficos, lo que equivale a proporcionar una respuesta azarosa. Este tipo de comportamiento persiste a lo largo del juego, y evidencia que el niño no comprende su dinámica, o que no es capaz de realizar un razonamiento deductivo: 
*MOT: hum@i pero pregunta cosas para: adivinarlo .

*CHI: no lo sé: [=! hand over his mouth].

Las transgresiones de calidad y relación, en sus diversos tipos, son déficits pragmáticos específicos que se manifiestan también en otros síndromes genéticos neuroevolutivos que cursan con discapacidad intelectual (Fernández-Urquiza et al., 2015c, 2017).

\subsection{DÉFICIT PRAGMÁTICO DE BASE GRAMATICAL}

Además de déficits pragmáticos específicos, en el corpus CHROMOLANG encontramos también déficits de base gramatical, es decir, problemas comunicativos que tienen su origen en alteraciones en los componentes fonológico, morfosintáctico y léxico.

Desde el punto de vista pragmático, estas alteraciones pueden interpretarse como transgresiones de modo o manera (MMN), que con frecuencia derivan en transgresiones de cantidad (MQT), ya que las dificultades expresivas suelen ir ligadas a un discurso poco eficaz que habitualmente provoca una reducción del contenido informativo, como se observa en el siguiente ejemplo, en el que CHROMOLANG_2 trata de explicar un ejercicio de suma que tuvo que hacer en la escuela:

*ADU: a ver@i cuál era la tarea cuéntanos .

${ }^{*} \mathrm{CHI}: \quad$ e:la $\left[{ }^{*}\right][/]$ ela $\left[{ }^{*}\right]$ poner xxx xxx poniendo uno:s [/] unos eulos [*] y otro [*] eulos [*].

\%err: ela=era $\$ \mathrm{PHO}$; ela=era $\$ \mathrm{PHO}$; eulos=euros $\$ \mathrm{PHO}$; otro=otros $\$ \mathrm{MOR}$

\%xepr: \$15:MMN:REP; \$15:MMN:REP; \$15:MMN:ORD:OMI; \$i5:MQT:VUT

*CHI: y: [/]<y: pa:(ra)@d> [/] y pa:@d hacer xxx xxx <y habia> [/] y < habia como:> [/] había co:mo: [/] como billetes y eulos [*] y ya a:h debajo [=! deictic illustrator] billetes y euros

\%err: $\quad$ eulos=euros $\$ \mathrm{PHO}$

\%xepr: \$i5:MMN:REP; \$i5:MMN:REP; \$i5:MMN:REP; \$i5:MMN:REP; \$i5:MMN:REP; \$i5:MQT:RUT; \$15:MQT:EVP

Se observa que las repeticiones (REP) y las omisiones (OMI) de morfemas, unidos a la falta de inteligibilidad, dan como resultado un primer enunciado vago (VUT), mientras que el segundo enunciado resulta redundante (RUT), y la producción verbal excesiva (EVP) en relación con el contenido que consigue transmitir, algo que ocurre también en el ejemplo siguiente:

*ADU: y qué hace tu hermana en Alemania ?

${ }^{*}$ CHI: tabajá $[*]$.

\%err: tabajá=trabajar $\$$ PHO

*ADU: trabaja en Alemania?

${ }^{*} \mathrm{CHI}: \quad 0$ [=! nods $]$. [+ trn]

*ADU: y por qué se ha ido tan lejos ?

${ }^{*} \mathrm{CHI}$ po:s@d para: [/-] (.) <para que:> [/] pala [*] que: <pued(a) i(r) a> [?] tabajá [*] xxx .

\%err: $\quad$ pala=para $\$$ PHO; tabajá=trabajar $\$$ PHO

\%xepr: \$i5:MMN:REF; \$i5:MMN:REP; \$i5:MQT:RUT; \$i5:MQT:EVP 
En otros casos las respuestas resultan redundantes por ecolálicas (ECO). Si bien la ecolalia es un fenómeno que evidencia problemas de comprensión, simultáneamente pone de manifiesto la voluntad del niño de contribuir al intercambio comunicativo mediante la construcción de turnos colaborativos, como en el siguiente ejemplo [CHROMOLANG_2]:

*MOT: la síla:(ba) +//.

${ }^{*} \mathrm{CHI}: \quad++$ la zila .

\%err: $\quad$ zila=silaba \$PHO

\%xepr: \$i5:MQT:RUT:ECO:ICOM

*MOT: la silaba qué ?

${ }^{*} \mathrm{CHI}: \quad++$ tónica .

\subsection{CONDUCTAS COMPENSATORIAS}

Ejemplos como el anterior deben hacernos pensar en la importancia de interpretar ciertas conductas que normalmente se han calificado como deficitarias en clave de estrategias compensatorias de las dificultades expresivas que el niño experimenta. Entre estas estrategias se encuentra el uso de interjecciones (INT) que generan implicaturas particularizadas (PIM) y que sustituyen a la producción verbal explícita, como se observa en el ejemplo siguiente, en el que la respuesta del interlocutor revela que ha captado el contenido implícito que CHROMOLANG_2 pretendia transmitir:

*MOT: y cuántos hermanos tiene papá ?

${ }^{*} \mathrm{CHI}$ : $\quad$ prff@i [=! looks away] .

\%xepr: \$i5:MQT:RVP:INT; \$et3:GSA; \$i5:PIM:PMQT:ADQ

*ADU: sí (.) tantos tiene ?

Muy a menudo estas interjecciones se acompañan de gestualidad compensatoria (GSA), de manera que las implicaturas se generan por medios paraverbales y no verbales, como en el siguiente ejemplo de CHROMOLANG_3, una niña de 10;2 años en el momento de la grabación y con una microduplicación en $1 \mathrm{q} 42.3 \mathrm{q} 43$. La niña responde a la pregunta de su madre con una interjección y un gesto deíctico, y así comunica implícitamente que va a dormir con su hermano, quien capta la implicatura y bromea al respecto:

*MOT: y dónde vas a dormir?

${ }^{*} \mathrm{CHI}: \quad$ ah@i [=! points to BOY] .

\%xepr: \$15:MQT:RVP:INT; \$15:PIM:PMQT:ADQ; \$et3:GSA

*BOY: en la cama de arriba [?] con la abuela [=! joking] .

*GMA: ea@i conmigo !

${ }^{*} \mathrm{CHI}: \quad 0$ [=! keeps pointing to BOY]. [+ trn]

\%xepr: \$et3:GSA; \$i5:PIM:PMQT:ADQ

*BOY: <conmigo no> [!] yo duermo en una cama aparte .

La generación de implicaturas constituye una conducta compensatoria especialmente eficaz en los casos en que no hay déficit pragmático específico 
y el déficit de base gramatical es muy acusado, como ocurre con CHROMOLANG_3. La siguiente conversación se produce cuando la niña le entrega a su padre una tarjeta que ha hecho para él en el colegio:

*FAT: ábrela .

${ }^{*} \mathrm{CHI}$ : va:le@i [=! starts opening the envelope] <tuya e:> [=! shouting] .

\%xepr: \$i5:PIM:PMQT:ADQ

*FAT: vale@i pero léemela!

${ }^{*} \mathrm{CHI}$ : va:le@i .

La niña transgrede ostensivamente la máxima de cantidad al emitir un enunciado escasamente informativo (tuya es), que incide sobre una información mutuamente manifiesta (que la tarjeta es para su padre). Al hacerlo, implica adecuadamente que es su padre quien deberia abrir la tarjeta, implicatura que es captada por su progenitor, como evidencia su respuesta.

Más interesante aún es el hecho de que CHROMOLANG_3 es capaz de captar implicaturas débiles en contextos considerablemente más complejos. Así, por ejemplo, durante una conversación familiar sobre los regalos que la niña pediría por su comunión, el resto de participantes (sus abuelos, su padre, su hermano) bromea con la niña diciendo cosas que no son ciertas y que es mutuamente manifiesto para todos que no lo son, es decir, transgrediendo ostensivamente la máxima de calidad, como en el siguiente ejemplo:

*MOT: qué te gusta a ti?

*FAT: la Play+Cinco [=! joking] .

${ }^{*} \mathrm{CHI}$ : no (.) apoco $\left[{ }^{*}\right][=!$ turns to FAT, taps him in the arm, smiles] .

\%err: apoco=tampoco $\$ \mathrm{PHO}$

\%xepr: \$i5:PIM:PMQL:ACOM

La respuesta relajada de la niña, que se gira hacia su padre, le sonrie y le da un golpecito en el brazo, pone de manifiesto que capta la comicidad de su intervención, es decir, capta la actitud proposicional de su enunciado o, en otras palabras, que su padre no habla en serio. Y es más, CHROMOLANG_3 es también capaz de generar implicaturas utilizando este mismo mecanismo, lo que requiere no solo buena capacidad metarrepresentacional, sino también la habilidad de desligarse de los usos literales del lenguaje, es decir, la capacidad de emitir enunciados que no pretenden comunicar la proposición que codifican, lo que sin duda no puede hacer un hablante con déficit pragmático específico. En el ejemplo siguiente, la niña dice que quiere un gato como regalo para su comunión, esperando que su familia se dé cuenta de que no es cierto, que ella también está de broma:

${ }^{*} \mathrm{CHI}: \quad$ no (.) a:h una: [/-] un ga:to .

\%xepr: \$i5:PIM:PMQL:ADQ

*MOT: un gato [/] un gato ?

${ }^{*}$ CHI: $\quad$ sí [=! nods, smiles] .

*GMA: <lo que nos faltaba ya también> [?] .

${ }^{*} \mathrm{CHI}$ : es mentira es mentira [=! showing $\left.\mathrm{EBM}\right]$. 
Entre las conductas compensatorias más utilizadas por nuestros informantes encontramos también las expresiones que ponen de manifiesto la conciencia de su dificultad expresiva (del tipo no sé, no me acuerdo, ¿cómo se llama?), que suelen ir acompañadas de contacto visual directo con el interlocutor, y constituyen un acto explícito de petición de ayuda (ERQ) [CHROMOLANG_2]:

*MOT: y de qué se opera „Joaquín ?

${ }^{*} \mathrm{CHI}:$ de: [/] de: <creo: de:> [/-] no sé [=! looks at MOT] .

\%xepr: \$15:MMN:REP; \$15:MMN:REF; \$et3:DRA:ERQ

Hay que tener en cuenta que este tipo de enunciados no solo rellenan el turno, sino que informan al interlocutor de cómo puede colaborar para que la conversación avance con éxito, por lo que siempre son preferibles a las pausas largas en el interior del turno o al silencio.

Otro tipo de conducta que puede interpretarse como una estrategia compensatoria del déficit de base gramatical es la perseveración temática (PER), cuando se produce en ciertos contextos. Así, por ejemplo, cuando las dificultades expresivas son severas y el grado de inteligibilidad del discurso del informante es muy bajo, puede ocurrir que el interlocutor no tematice los enunciados del niño, y que intente desarrollar otros temas para seguir adelante con el intercambio. Esto es lo que ocurre en el siguiente fragmento de conversación entre CHROMOLANG_1 y su madre:

*MOT: tenemos que ponerles unos precio:s buenos

${ }^{*} \mathrm{CHI}: \quad$ ete $\left[{ }^{*}\right]$ \&n \&a: ande $\left[{ }^{*}\right] \&$ d eta $\left[{ }^{*}\right]<$ una o dos> [?] [=! looks MOT in the eyes] .

${ }^{*} \mathrm{CHI}: \quad$ ete $\left[{ }^{*}\right]<\&$ onda $[/]$ nde $>\left[{ }^{*}\right]$ ta $\left[{ }^{*}\right]$.

\%err: ete=este $\$ \mathrm{PHO}$; nde=dónde $\$ \mathrm{PHO}$; ta=está $\$ \mathrm{PHO}$

\%xepr: \$et3:DRA:DIS; \$i5:MRL:QST:PER

*MOT: venga@i qué precio <les vamos a poner $>$ [>] ?

${ }^{*} \mathrm{CHI}$ : nde [*] ta [*] [<] [=! shouting, looks up to the shelves searching for something] .

\%err: $\quad$ nde=dónde \$PHO; ta=está \$PHO

\%xepr: \$i5:MRL:QST:PER

${ }^{*} \mathrm{CHI}: \quad$ ete $\left[{ }^{*}\right]$ \&n ande $\left[{ }^{*}\right]$ ta $\left[{ }^{*}\right]<$ na da: $>$ [?].

\%err: ete=este \$PHO; ande=dónde \$PHO; ta=está \$PHO

\%xepr: \$et3:DRA:DIS; \$15:MRL:QST:PER

Madre e hijo se encuentran solos en la habitación, jugando con los coches que hay encima de la mesa. La madre propone jugar a ordenar los coches y ponerles precio, pero el niño coge un coche grande y pregunta repetidamente algo sobre él. Los enunciados del niño resultan ininteligibles, debido a las omisiones de sonidos tanto en margen prenuclear como postnuclear de sílaba, y en sílabas tanto átonas como tónicas. A estas omisiones se unen importantes disfluencias que alteran la curva melódica del enunciado, cambios en el volumen de voz y, en general, un gran esfuerzo articulatorio. La madre, incapaz de comprender lo que dice su hijo, ignora la pregunta del niño en dos ocasiones, lo que provoca las versiones de insistencia de CHROMOLANG_1 en su empeño por hacerse entender. Sin duda, esta conducta 
es atípica en un niño de la edad de CHROMOLANG_1 (5;8 años), en el sentido de que no parece consciente de la ininteligibilidad de sus emisiones, lo que denota una ausencia de conciencia metapragmática. Sin embargo, no es una conducta anómala desde el punto de vista comunicativo, puesto que la perseveración no es inmotivada, sino que pone de manifiesto la voluntad del niño de continuar con el intercambio y ver satisfecha su demanda informativa, en lugar de resignarse y continuar con el juego.

Finalmente, el uso del estilo directo (RPS:DIR) para escenificar anécdotas personales permite a los informantes del corpus CHROMOLANG evitar el coste de procesamiento extra que supondría el uso del estilo indirecto desde el punto de vista morfosintáctico y, en este sentido, puede considerarse también una estrategia compensatoria del déficit gramatical:

${ }^{*} \mathrm{CHI}: \quad$ hacía asto $[*]+" /$.

\%err: asto=esto $\$ \mathrm{PHO}$

*CHI: +" bla bla bla bla bla bla bla bla.

${ }^{*} \mathrm{CHI}: \quad$ y Don+Juan como: +"/.

${ }^{*} \mathrm{CHI}: \quad+$ palalos [*] ya [=! hits the table imitating his teacher] .

\%err: palalos $=$ pararos $\$ \mathrm{PHO}$

\section{Conclusiones}

La evaluación pragmática del lenguaje oral de niños con cromosomopatías de baja prevalencia en conversación espontánea con sus familiares cercanos puede evidenciar fenómenos que las baterías tradicionales de test para la evaluación de los componentes estructurales del lenguaje no están preparadas para detectar.

En este trabajo hemos defendido que la valoración de la competencia comunicativa del niño, es decir, del uso que es capaz de hacer de sus recursos lingüísticos preservados, es fundamental para completar su diagnóstico, ya que nos permite evaluar no solo los déficits estructurales del lenguaje del sujeto, sino también sus disfuncionalidades pragmáticas. Al mismo tiempo, hemos insistido en la necesidad de prestar atención a las fortalezas funcionales, es decir, a las conductas verbales, paraverbales, y no verbales que despliega el niño de manera espontánea y que constituyen estrategias compensatorias del déficit de base gramatical.

La evaluación pragmático-funcional que proponemos se implementa por medio de una metodología en la que se integran tres elementos clave: 1) el carácter cualitativo de los estudios pioneros en el ámbito de la Lingüística Clinica (presente en instrumentos como el PREP-R); 2) las herramientas tecnológicas del proyecto CHILDES, que posibilitan la transcripción y codificación estandarizadas de la muestra, así como la realización de recuentos automáticos una vez analizados los datos, y 3) herramientas de etiquetado pragmático específicamente diseñadas para el análisis de corpus clínicos de lenguaje oral (PREP-CORP) de sujetos con síndromes genéticos neuroevolutivos. 
El análisis pragmático resultante de la aplicación de esta metodología permite afinar la especificidad de la intervención logopédica, orientándola hacia los aspectos lingüísticos, paralingüísticos, no verbales, o cognitivos que sea necesario modificar o potenciar para que el niño pueda comunicarse con éxito, a pesar de que puedan persistir los déficits estructurales en su lenguaje.

\section{REFERENCIAS}

BENÍTEZ-BURRACO, A. et al. (2018): "Narrowing the Genetic Causes of Language Dysfunction in the 1q21.1 Microduplication Syndrome", Frontiers in Pediatrics, 6, pp. 1-15.

BENÍTEZ-BURRACO, A. et al. (2018): "Language impairment in a boy with a microdeletion in $8 \mathrm{p} 23.1$ and a microduplication in 9p24.3", V Congreso Internacional de Lingüística Clínica, Cádiz, del 7 al 9 de noviembre de 2018.

BENÍTEZ-BURRACO, A. et al. (2018): "Language impairment in a girl with a microduplication in 1q42.3q43", V Congreso Internacional de Lingüistica Clínica, Cádiz, del 7 al 9 de noviembre de 2018.

CRYSTAL, D. (1981): Clinical Linguistics, London: Whurr Publishers.

CRYSTAL, D. (1982): Profiling Linguistic Disability, London: Edward Arnold.

DAVIS, G. (2007): "Cognitive Pragmatics of Language Disorders in Adults", Seminars in Speech and Language, 28 (2), pp. $111-$ 121.

DÍAZ MARTÍNEZ, F. (1995): "Algunos aspectos de organización conversacional en consultas de logopedia”, Gallardo-Paúls, B., Hernández, C. y Moreno, V. (eds.); Lingüistica clínica y neuropsicología cognitiva. Actas del Primer Congreso Nacional de Lingüística Clínica. Lingüistica interaccional en ámbitos de salud, Valencia: Universidad de Valencia, Volumen 3, pp. 1730.

DIEZ-ITZA, E. et al. (2014): "The Syndroling Project: A comparative linguistic analysis of typical development profiles and neurodevelopmental genetic syndromes (Down, Williams and Fragile X syndromes). Conclusions: Syndrome-specific profiles", IASCL-XII International Congress for the Study of Child Language, Ámsterdam, del 14 al 18 de julio de 2014 .

DIEZ-ITZA, E.; SNOW, C. E., y MACWHINNEY, B. (1999): "La metodologia RETAHME y el proyecto CHILDES: Breviario para la codificación y análisis del lenguaje infantil", Psicothema, 11, pp. 517-530.

DONNAI, D. \& KARMILOFF-SMITH, A. (2000): "Williams syndrome: from genotype through to the cognitive phenotype", American Journal of Medical Genetics, 97, pp. 164-71.

EKMAN, P. \& FRIESEN, W. V. (1981): "The repertoire of nonverbal behavior: Categories, origins, usage, and coding", Kendon, J., Sebeok, T. A., \& UmikerSebeok, J. (eds.): Nonverbal communication, interaction, and gesture. Selections from SEMIOTICA, Boston: Mouton de Gruyter, pp. 57-106. 
FERNÁNDEZ PÉREZ, M. (2000): “El enfoque pragmático en el diseño y proyección de pruebas de evaluación lingüística en edad infantil", Pragmalingüistica, 8, pp.113-122.

FERNÁNDEZ-URQUIZA, M. et al. (2015a): PREP-R. Protocolo Rápido de Evaluación Pragmática Revisado, Valencia: Universidad de Valencia.

FERNÁNDEZ-URQUIZA， M. y GALLARDO-PAÚLS, B. (2015b): "Adaptación del formato CHAT para la transcripción y codificación de los datos de lenguaje patológico del corpus PerLA", Oralia, 18, pp. 81-102.

FERNÁNDEZ-URQUIZA, $\mathrm{M}$. et al. (2015c): "Perfiles pragmáticos comparados de sindromes genéticos neuroevolutivos (S. Williams, S. Down y S. X Frágil)", Diéguez-Vide, F. (ed.): Temas de Lingüistica Clinica, Barcelona: Horsori Editorial, pp. 89-90.

FERNÁNDEZ-URQUIZA, M.; DIEZITZA, E. y CORTIÑAS, S. (2017): "PREP-CORP: Sistema de etiquetado pragmático de corpus clínicos de lengua oral", Fernández López, M. C.; Martí Sánchez, M. y Ruiz Martínez, A. M. (eds.): Investigaciones actuales en lingüistica. Vol. VI: Aplicaciones de la Lingüística, Alcalá de Henares: Universidad de Alcalá, pp. 167-183.

GALLARDO-PAÚlS, B. (2007): Pragmática para logopedas, Cádiz: Servicio de Publicaciones de la Universidad de Cádiz.

GALLARDO-PAÚLS, B. (2009): "Valoración del componente pragmático a partir de datos orales", Revista de Neurología, 48 (2), pp. S57-S61.

GRICE, H. P. (1975): "Logic and Conversation", Cole, J. \& Morgan P. (eds.): Syntax and Semantics. Volume 3: Speech Acts, Nueva
York: Academic Press, pp. 4158.

JAKOBSON, R. (1935): "Aphasia as a linguistic problem", Werner, $\mathrm{H}$. (ed.): On Expressive Language, Worcester, MA: Clark University Press, pp. 69-81.

JAKOBSON, R. (1941): Child Language, Aphasia, and Phonological Universals, The Hague: Mouton de Gruyter, 1968.

JAKOBSON, R. (1956): "Two aspects of language and two types of aphasic disburbances", Jakobson, R. \& Halle, M. (eds.): Fundamentals of Language, The Hague: Mouton de Gruyter, pp. 50-82.

LESSER, R. (1978): Investigaciones lingüisticas sobre la afasia, Barcelona: Editorial Médica y Técnica, 1983.

MACWHINNEY, B. (2000): The CHILDES Project: Tools for Analyzing Talk. Volume I: Transcription format and programs, Mahwah, NJ: Lawrence Erlbaum.

MACWHINNEY, B. \& SNOW, C. (1985): "The Child Language Data Exchange System", Journal of Child Language, 12, pp. 271-296.

MACWHINNEY, B. \& SNOW, C. (1990): "The Child Language Data Exchange System: An update", Journal of Child Language, 17, pp. 457-472.

MUÑOZ-CÉSPEDES, J. M. y MELLE, N. (2004): "Alteraciones de la pragmática de la comunicación después de un traumatismo craneoencefálico", Revista de Neurología, 38 (9), pp. 852-859.

POYATOS, F. (2003): "Comunicación No Verbal: Algunas de sus perspectivas de estudio e investigación", Revista de Investigación Lingüistica, VI (2), pp. 67-83.

VERSCHUEREN, J. (1999): Understanding pragmatics, London: Edward Arnold. 